\title{
BMJ Open Impacts on health outcomes and on resource utilisation of home-based parenteral chemotherapy administration: a systematic review protocol
}

Benedicte Mittaine-Marzac, , ${ }^{1,2}$ Matthieu De Stampa,, ${ }^{2,3}$ Emmanuel Bagaragaza,,4 Joël Ankri, ${ }^{2,5}$ Philippe Aegerter ${ }^{2,6}$

To cite: Mittaine-Marzac B, De Stampa M, Bagaragaza E, et al. Impacts on health outcomes and on resource utilisation of homebased parenteral chemotherapy administration: a systematic review protocol. BMJ Open 2018;8:e020594. doi:10.1136/ bmjopen-2017-020594

- Prepublication history and additional material for this paper are available online. To view these files, please visit the journal online (http://dx.doi. org/10.1136/bmjopen-2017020594).

Received 10 November 2017 Revised 9 March 2018 Accepted 13 March 2018
Check for updates

For numbered affiliations see end of article.

\section{Correspondence to} Dr Benedicte Mittaine-Marzac; benedicte.mittaine-marzac@ aphp.fr

\section{ABSTRACT}

Introduction Despite the demonstrated feasibility and policies to enable more to receive chemotherapy at home, in a few countries, parenteral chemotherapy administration at home remains currently marginal. Of note, findings of different studies on health outcomes and resources utilisation vary, leading to conflicting results. This protocol outlines a systematic review that seeks to synthesise and critically appraise the current state of evidence on the comparison between home setting and hospital setting for parenteral chemotherapy administration within the same high standards of clinical care.

Methods and analysis This protocol has been prepared following the Preferred Reporting Items for Systematic Reviews and Meta-Analyses Protocols approach. Electronic searches will be conducted on bibliographic databases selected from the earliest available data through 15 November 2017 published in French and English languages. Additional potential papers in the selected studies and grey literature will be also included in the review. The review will include all types of studies exploring patients receiving anticancer drugs for injection at home compared with patients receiving the drugs in a hospital setting, and will assess at least one of the following criteria: patients' health outcomes, patients' or caregivers' satisfaction, resource utilisation with cost savings, and incentives and/or barriers of each admission setting according to patients' and relatives' points of view. Two reviewers will independently screen studies and extract relevant data from the included studies. Methodological quality of studies will be assessed using the 'Quality Assessment Tool for Quantitative Studies' developed by the Effective Public Health Practice Project tool, in addition to the Consolidated Health Economic Evaluation Reporting Standards statement for economic studies.

Ethics and dissemination As the review is focused on the analysis of secondary data, it does not require ethics approval. The results of the study will be disseminated through articles in peer-reviewed journals and trade publications, as well as presentations at relevant conferences.

PROSPERO registration number CRD42017068164.

\section{INTRODUCTION}

Hospitals remain the main setting for parenteral cancer chemotherapy administration

\section{Strengths and limitations of this study}

- This will be one of the first attempts to synthesise and critically appraise the current state of evidence on the comparison between home setting and hospital setting for parenteral chemotherapy administration.

- This systematic review protocol is developed using the Preferred Reporting Items for Systematic Reviews and Meta-Analyses Protocols guidelines.

- Based on previous work, we anticipate methodological heterogeneity and statistical heterogeneity, leading to difficulty in analysing data quantitatively.

- The definition of home-based services including different types of interventions is confusing, leading to discrepancies when analysing.

despite patients' generally positive feeling about receiving care at home. ${ }^{1}$ As the worldwide incidence of cancers increases, ${ }^{2}$ and bearing in mind that due to progress in the efficacy of treatments cancer is becoming a long-term disease, along with advances in diagnostic technology and novel targeted treatments, and due to financial constraints, it is necessary to develop alternative models of health service delivery, such as home programmes, without detrimental effects on health outcomes and costs. Despite the feasibility $^{3-5}$ and despite policies to enable more to receive chemotherapy at home, in a few countries, ${ }^{67}$ parenteral chemotherapy administration at home remains marginal due to little evidence on improving health outcomes, patients' quality of life and cost savings. Furthermore, findings on outcomes varied, leading sometimes to conflicting results. ${ }^{8-10}$ A recent scoping study demonstrates that most economic studies concluded in favour of home care, but according to the appropriate method of assessment, all sources of expenditure (specialised clinical 
care, transportation, out-of-pocket expenses and so on) were not systematically assessed, ${ }^{11}{ }^{12}$ whereas other studies showed no significant difference in the overall costs. ${ }^{9} 10$ Likewise, the impact on caregivers, especially the shift of the care burden from the hospital to relatives, has rarely been studied. To date, there is only one existing review of home-based chemotherapy ${ }^{13}$ and it ended in favour of administration at home, but this conclusion is hampered by a mix between routes of administration (parenteral and oral intake) and type of care (administration and postadministration follow-up).

Considering these facts, a systematic review will be undertaken to provide a complete overview of parenteral cancer chemotherapy administration at home to investigate whether the clinical outcomes are maintained and resource utilisation is reduced in comparison with hospitals' high standards of care.

\section{METHODS}

This protocol has been prepared following the Preferred Reporting Items for Systematic Reviews and Meta-Analyses Protocols (PRISMA-P) approach ${ }^{14}$ to increase confidence in the findings. It has been registered in the International Prospective Register of Systematic Reviews database (trial registration number: CRD42017068164).

\section{Types of studies}

Except crossover studies, all types of studies that have investigated parenteral cancer chemotherapy home administration compared with hospital setting, such as randomised controlled or uncontrolled trials, cohort studies and parallel studies, will be included if they fulfil at least one of the following criteria:

1. Assessment of the impact of parenteral chemotherapy administration on patients' health outcomes whatever they are: survival, relapse and tolerance.

2. Assessment of the impact of parenteral chemotherapy administration on patients' or caregivers' satisfaction.

3. Assessment of the impact of parenteral chemotherapy administration on cost savings.

4. Assessment of the incentives and/or the barriers according to patients' and/or relatives' points of view.

Economic evaluations of resource utilisation with costs and consequences such as cost-effectiveness, cost utility or cost-benefit, either with or without any clinical assessment, will be included.

Crossover studies will be excluded from the review; crossover studies involving oncology products or the type of hospitalisation setting could suffer from order effects, so it could be difficult to confirm statistically the lack of order effects and hence to use the results.

\section{Participants}

We will be interested in studies on participants receiving parenteral anticancer drug administration at home compared with patients receiving the drugs in a hospital setting. There will be no restriction on the type of tumour disease, age or sex of participants.

\section{Patients and public involvement}

As the review is focused on the analysis of secondary data, it does not involve patients, nor does it involve patients in the design of the study or in the dissemination of results.

\section{Eligibility criteria}

All studies exploring parenteral anticancer drug administration at home setting, mainly intravenous and subcutaneous administrations, will be selected from the earliest available data through 15 November 2017, published in French and English languages, regardless of country. The inclusion criterion defining the scope of publications included in the review will be the comparison of parenteral chemotherapy administration at home with administration in inpatients and/or outpatients wards. As our goal is to study the home-based setting as an alternative context for providing the highest standards of clinical care concerning parenteral drug delivery, we will exclude all community-based services with no anticancer drug administration at home, and studies targeting only postadministration chemotherapy monitoring, disconnection of portable diffuser, management of central venous catheter and supportive care therapy. For the same reasons, studies on oral anticancer drugs will be excluded as the oral treatment does not require hospital care; patients usually manage their pills themselves with an ambulatory follow-up and the treatment issues are deeply different (ie, chronic side effects, patients' compliance).

\section{Databases and search strategy}

A detailed literature search will be conducted to identify all articles studying chemotherapy administration in a home setting for adults and paediatric patients. Published studies will be identified through searches of the Medline, Cochrane, Web of Science, Embase and Cumulative Index to Nursing and Allied Health from the earliest available date through 15 November 2017. HAH is defined as the delivery of hospital ward-level care and replaces the hospital by ensuring the continuity of care for the patient it takes care of. HAH is also known as 'hospital in the home', 'home hospitalization' and 'early supported discharge'. ${ }^{15-17}$ According to Medical Subject Headings (MeSH) definition, 'Home Care Services' is a community health and nursing service providing coordinated multiple services to patients at their homes. These home care services are provided by a visiting nurse, home health agencies, hospitals or organised community groups using professional staff for care delivery. They differ from home nursing, which is provided by non-professionals, and from 'Home Care Agencies', public or private organisations, which provide home health services in patients' home either directly or through arrangements with other organisations.

Therefore, we have developed a strategy in Medline to retrieve relevant literature on the topic. The Medline 
search strategy will be ("Neoplasms" [Mesh] AND ("Antineoplastic Combined Chemotherapy Protocols" [Mesh] OR "Antineoplastic Agents"[Mesh])) AND ("Home Care Services"[Mesh] OR "Home Care Agencies"[Mesh]). This search strategy will be adapted in searching other databases (see search strategy in online supplementary file). The references from potentially relevant papers were manually searched for additional studies. A further research will be performed in OpenGrey, in Research Papers in Economics and in Google Scholar databases to find relevant unpublished studies in grey literature.

\section{Study selection}

We will use a two-stage process to identify papers that met our inclusion criteria. The first stage accumulated all papers from two types of searches using the already mentioned databases. The first type of search will use combinations of keywords to retrieve titles of potentially relevant studies. The search output will be screened independently by two review authors (MDS, BM-M) to identify studies that potentially meet the inclusion criteria outlined above. In case of discrepancy, the abstract of the concerned study will be retrieved. If disagreements remained, a third author (EB) will arbitrate. In the second type of search, all papers citing or cited by articles that had already met our inclusion criteria will be identified, then screened as the first ones. In the second stage, the full text of these selected studies will be retrieved and independently assessed for eligibility by the same two review members. An article will be considered relevant and kept for analysis if the full text of the publication is of a comparison design assessing patients' health and/ or safety outcomes and/or patients' or relatives' satisfaction, and/or cost savings and/or the incentives and the barriers of using home as an alternative setting. Duplicates of articles will be discarded.

Any disagreement between the reviewers over the eligibility of particular studies will be resolved through a discussion with a third reviewer (EB).

\section{Data extraction}

Extracted information on a standardised form will include the following:

- Study setting.

- Study population.

- Tumour type and chemotherapy regimen.

- Details of the intervention/control conditions.

- Experimental design.

- Recruitment and study completion rates.

- Patients' health outcomes including quality of life, duration of survival, progression of disease and toxicity.

- Patients' and caregivers' satisfaction and preference, and shift of burden.

- Resource utilisation with costs and consequences including cost-effectiveness, cost utility or cost-benefit analyses, and perspective of the study including provider, payer or societal and shift of costs.
- Incentives and barriers according to patients' and/or relatives' points of view.

- Information for the assessment of the risk of bias.

Data will be extracted independently by two review members; discrepancies will be identified and resolved through a discussion with a third reviewer (PA).

\section{Data assessment and synthesis}

\section{Quality assessment}

Two reviewers (MDS and BM-M) will independently assess the quality of included studies and the potential risk of bias. We will use the 'Quality Assessment Tool for Quantitative Studies' developed by the Effective Public Health Practice Project tool, ${ }^{18}$ as it can be applied to articles of any public health topic area, assessing the components of studies (design, methods, bias). Likewise, we will use the Consolidated Health Economic Evaluation Reporting Standards statement, ${ }^{19}$ focusing on the design quality of the cost studies. It leads to an overall methodological rating of strong, moderate or weak. In case of disagreement, consensus will be reached through a discussion.

\section{Data synthesis}

According to the limited development of home parenteral chemotherapy administration, we anticipate methodological heterogeneity, statistical heterogeneity, heterogeneity of interventions and heterogeneity of models against a meta-analysis of the evidence base. In that case, an interpretative method should be more appropriate, leading to a description of the observed effects with peculiarities, and strengths and limits of each study, according to the quality of the study, type of study, studied population and interventions. The Grading of Recommendations Assessment, Development and Evaluation approach ${ }^{20}$ will be used to assess the overall quality of evidence by downgrading scores in case of serious risk of bias, imprecision and study limitations, or indeed by upgrading scores of studies that traditionally have been considered to have a weak design. It would be appropriate to assess relevant studies that do not provide evidence on every outcome. Final scores on the quality of evidence will be categorised as high, moderate, low or very low, and summarised in a table. This method should be more appropriate from a holistic point of view.

\section{Subgroup analysis}

If the data are available, we will conduct subgroup analyses investigating the effects of study design, models, interventions, tumour type (haematological tumour, solid tumour) and age of the study population (children, young people $<18$ years old, adults, $>65$ years old).

A sensitivity analysis will determine the robustness of the observed outcomes facing publication language, clinical or methodological heterogeneity.

\section{Ethics and dissemination}

As the review is focused on the analysis of secondary data, it does not require ethics approval. The results of the study will be disseminated through articles in peer-reviewed 
journals and trade publications, as well as presentations at relevant conferences.

\section{CONCLUSION}

This review, following the PRISMA-P guidelines, will be an attempt to synthesise and to critically appraise the impact of parenteral chemotherapy administration at home for patients and their relatives compared with a hospital setting.

Other strengths of this review include the search of literature published in two languages, French and English, and the wide search from published studies in grey literature.

It is however acknowledged that the definition of home-based services including different types of interventions is confusing, which could lead to discrepancies during the analysis. Likewise, the results of the review may be limited by the diversity of study designs and present challenges in the quality assessment. These limitations will be considered in relation to the review findings.

However, this review could be useful for medical staff in choosing the best treatment setting for patients and their relatives, and could contribute to developing parenteral chemotherapy administration at home. As we anticipate great heterogeneity of the studies, mainly concerning the interventions, this review could at least highlight a definition of a realistic methodology to set up in this field.

\section{Author affiliations}

${ }^{1}$ Hospitalisation à Domicile Assistance Publique des Hôpitaux de Paris, Pharmacie à Usage intérieure, Paris, France

2UFR Médecine Paris-lle-de-France-Ouest Université Versailles St-Quentin, Unité Mixte de Recherche (UMR) 1168 INSERM, UVSQ, VIMA, Villejuif, France ${ }^{3}$ Hospitalisation At Home, Assistance Publique Hôpitaux de Paris, Paris, France ${ }^{4}$ Pôle Recherche SPES «Soins Palliatifs En Société», Maison Médicale Jeanne Garnier, Paris, France

${ }^{5}$ Hôpital Sainte Périne, Assistance Publique Hôpitaux de Paris, Paris, France ${ }^{6}$ Département de Santé Publique, Hôpital Ambroise Paré, Assistance Publique Hôpitaux de Paris, Unité de Recherche Clinique, Paris, France

Contributors All authors made substantive intellectual contributions to the development of this protocol. BM-M wrote this protocol. MDS, EB, PA commented critically on the manuscript and were involved in conceptualising the review. JA commented critically on the manuscript.

Funding This work is supported by the Hospitalisation At Home, Assistance Publique des Hôpitaux de Paris.

Competing interests None declared.

Patient consent Not required.

Provenance and peer review Not commissioned; externally peer reviewed.

Open Access This is an Open Access article distributed in accordance with the Creative Commons Attribution Non Commercial (CC BY-NC 4.0) license, which permits others to distribute, remix, adapt, build upon this work non-commercially, and license their derivative works on different terms, provided the original work is properly cited and the use is non-commercial. See: http://creativecommons.org/ licenses/by-nc/4.0/ (c) Article author(s) (or their employer(s) unless otherwise stated in the text of the article) 2018. All rights reserved. No commercial use is permitted unless otherwise expressly granted.

\section{REFERENCES}

1. Leff B, Burton L, Mader S, et al. Satisfaction with Hospital at Home Care. J Am Geriatr Soc 2016;54:1355-63.

2. Fitzmaurice C, Allen C, Barber RM, et al. Global, Regional, and National Cancer Incidence, Mortality, Years of Life Lost, Years Lived With Disability, and Disability-Adjusted Life-years for 32 Cancer Groups, 1990 to 2015: A Systematic Analysis for the Global Burden of Disease Study. JAMA Oncol 2017;3:524-48.

3. Anderson $\mathrm{H}$, Addington-Hall JM, Peake MD, et al. Domiciliary chemotherapy with gemcitabine is safe and acceptable to advanced non-small-cell lung cancer patients: results of a feasibility study. $\mathrm{Br} \mathrm{J}$ Cancer 2003;89:2190-6.

4. Polinski JM, Kowal MK, Gagnon M, et al. Home infusion: Safe, clinically effective, patient preferred, and cost saving. Healthc 2017;5:68-80.

5. Corbett M, Heirs M, Rose M, et al. The delivery of chemotherapy at home: an evidence synthesis. Health Services and Delivery Research 2015;3:1-182.

6. Hirtzlin I. Conditions du développement de la chimiothérapie en hospitalisation à domicile: analyse économique et organisationnelle, Haute Autorité de Santé. mise en ligne janvier 2015 https://www.hassante.fr/portail/jcms/c_1696038/fr/conditions-du-developpement-dela-chimiotherapie-en-hospitalisation-a-domicile.

7. Lüthi F, Fucina N, Divorne N, et al. Home care--a safe and attractive alternative to inpatient administration of intensive chemotherapies. Support Care Cancer 2012;20:575-81.

8. Borras JM, et al. Compliance, satisfaction, and quality of life of patients with colorectal cancer receiving home chemotherapy or outpatient treatment: a randomised controlled trial. BMJ 2001;322:826-5.

9. Stevens B, Croxford R, McKeever P, et al. Hospital and home chemotherapy for children with leukemia: a randomized cross-over study. Pediatr Blood Cancer 2006;47:285-92.

10. Corrie PG, Moody AM, Armstrong G, et al. Is community treatment best? a randomised trial comparing delivery of cancer treatment in the hospital, home and GP surgery. Br J Cancer 2013;109:1549-55.

11. Raphaël R, Yves D, Giselle C, et al. Cancer treatment at home or in the hospital: what are the costs for French public health insurance? Findings of a comprehensive-cancer centre. Health Policy 2005;72:141-8.

12. Lassalle $\mathrm{A}$, Thomaré $\mathrm{P}$, Fronteau $\mathrm{C}$, et al. Home administration of bortezomib in multiple myeloma is cost-effective and is preferred by patients compared with hospital administration: results of a prospective single-center study. Ann Oncol 2016;27:314-8.

13. Evans JM, Qiu M, MacKinnon M, et al. A multi-method review of home-based chemotherapy. Eur J Cancer Care 2016;25:883-902.

14. Moher D, Shamseer L, Clarke M, et al. Preferred reporting items for systematic review and meta-analysis protocols (PRISMA-P) 2015 statement. Syst Rev 2015;4:1.

15. Leff B, Burton L, Mader SL, et al. Hospital at home: feasibility and outcomes of a program to provide hospital-level care at home for acutely ill older patients. Ann Intern Med 2005;143:798-808.

16. Stessman J, Ginsberg G, Hammerman-Rozenberg R, et al. Decreased hospital utilization by older adults attributable to a home hospitalization program. J Am Geriatr Soc 1996;44:591-8.

17. Langhorne P, Taylor G, Murray G, et al. Early supported discharge services for stroke patients: a meta-analysis of individual patients' data. Lancet 2005;365:501-6.

18. National Collaborating Centre for Methods and Tools (2008). Quality Assessment Tool for Quantitative Studies. Hamilton, ON: McMaster University. Retrieved from. http://www.nccmt.ca/resources/search/14 (Updated 27 Jul 2017).

19. Husereau D, Drummond M, Petrou S, et al. Consolidated Health Economic Evaluation Reporting Standards (CHEERS)-explanation and elaboration: a report of the ISPOR Health Economic Evaluation Publication Guidelines Good Reporting Practices Task Force. Value Health 2013;16:231-50.

20. Balshem $\mathrm{H}$, Helfand $\mathrm{M}$, Schünemann $\mathrm{HJ}$, et al. GRADE guidelines: 3. Rating the quality of evidence. J Clin Epidemiol 2011;64:401-6. 